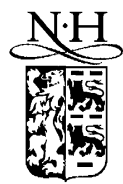

ELSEVIER

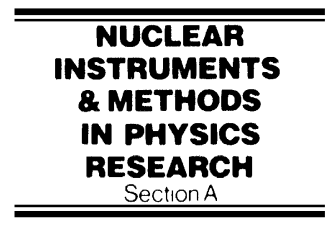

www.elsevier.com/locate/nima

\title{
Saturated logistic avalanche model
}

\author{
G. Aielli*, P. Camarri, R. Cardarelli, A. Di Ciaccio, B. Liberti, \\ A. Paoloni, R. Santonico \\ INFN and Dipartimento di Fisica dell'Università di Roma "Tor Vergata", Via della Ricerca Scientifica 1, Roma 00133, Italy
}

\begin{abstract}
The search for an adequate avalanche RPC working model evidenced that the simple exponential growth can describe the electron multiplication phenomena in the gas with acceptable accuracy until the external electric field is not perturbed by the growing avalanche. We present here a model in which the saturated growth induced by the space charge effects is explained in a natural way by a constant coefficient non-linear differential equation, the Logistic equation, which was originally introduced to describe the evolution of a biological population in a limited resources environment. The RPCs, due to the uniform and intense field, proved to be an ideal device to test experimentally the presented model.
\end{abstract}

(C) 2003 Elsevier Science B.V. All rights reserved.

PACS: $52.80 . \mathrm{Dy} ; 51.50 .+\mathrm{v} ; 29.40 . \mathrm{Cs}$

Keywords: Electron avalanche; Non-linear systems; RPC

\section{Non-linearity in the electron avalanche development}

The models basing on an exponential multiplication law can describe the electron avalanche in the gas with acceptable accuracy only in the limit in which the applied electric field, assumed to be a constant, is not perturbed by the growing avalanche [1]. This means that the probability per unit length of a ionizing collision for the free electrons does not depend on the presence of the other electrons. The falling of the previous requirement forces to abandon a comfortable linear description of the problem when the non-

\footnotetext{
*Corresponding author. Tel.: + 39-6-7259; fax: + 39-6-2023507.

E-mail address: aielli@roma2.infn.it (G. Aielli).
}

linearity is needed to describe the correlations between the electrons.

An usual approach to this problem [1,2] is based on the replacement of the exponential growth factor $\alpha$ with a chosen decreasing function $\alpha(x)$ to take into account the multiplication attenuation. Another possibility is the implementation of a finite elements calculation. This approach however would renounce to find the elements in terms of which the system is simply described; furthermore, having to do with strongly non-linear systems, as the avalanche to streamer transition region, the discretization presents the risk to introduce "artifacts". The investigation of interacting systems suggests a different approach, as strong correlation have one main consequence: they force the system to respond in a collective-like manner [3]. In this approach we will describe the electron 
avalanche in terms of a complex collective constituent object instead of the elementary charges, in term of which the system is described conveniently.

\section{The experimental base of the logistic model}

The avalanche growth in RPCs was extensively studied in Refs. [4-6], where the gas gain achievable with various gases was measured. A good experimental starting point, in view of understanding the avalanche development, are the plots showing how the prompt and total avalanche charges increase as a function of the applied voltage. All these plots exhibit an "exponential region" characterized by an exponentially growing charge, followed, at higher voltages, by a "saturation region" [4] where the charge grows at a weaker rate. In Fig. 1 the average prompt charge vs. the operating voltage for a gas containing small amounts of SF6 used as streamer suppressor [5] are plotted in a linear scale. The plot shows that the prompt charge increase in the saturation region is asymptotically linear. The total average charge behaves also in a similar way as shown in Fig. 2 for an RPC irradiated with $\gamma$ photons from a ${ }^{60}$ Co source $[7,6]$.

The experimentally observed growth of the avalanche charge recalls the Logistic function introduced firstly in 1845 by Verhulst [8] to

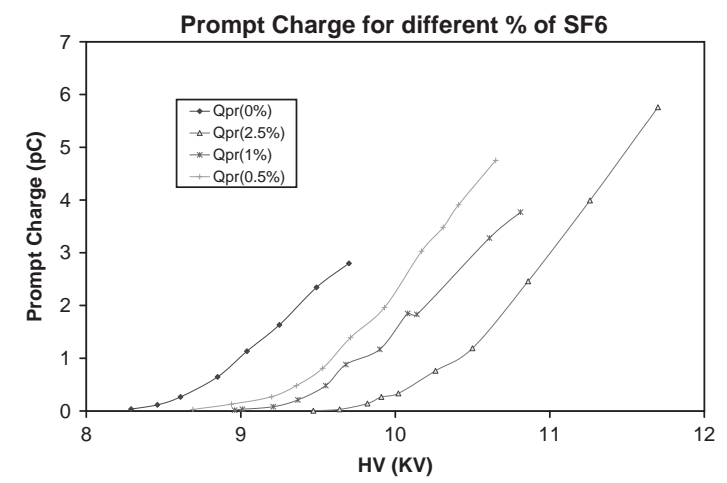

Fig. 1. Average prompt charge vs. applied voltage, for different amounts of $\mathrm{SF}_{6}$. The data are taken from 1 but plotted in linear scale.

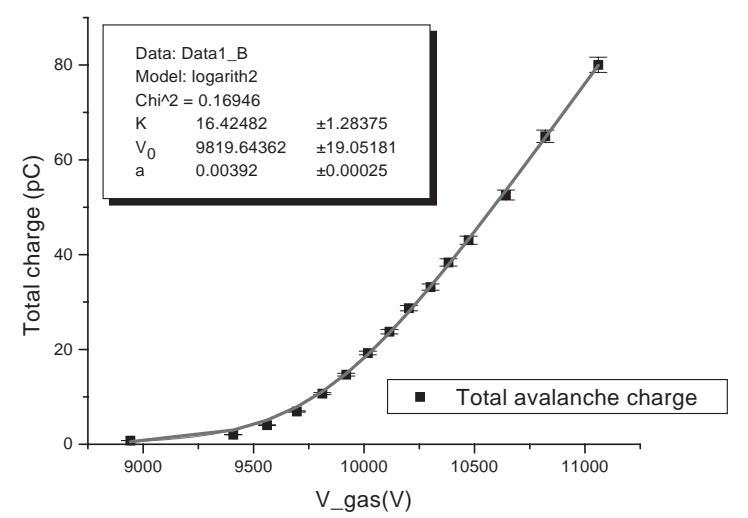

Fig. 2. Fit of the experimental points of figure with the logistic function's cumulative.

describe the growth of a biological population, that tend to become stationary in presence of a limited flow of food resources. In our case the experimental data on the total charge can be compared with the integral of the logistic function, accounting for the cumulative population. This can be written as a three parameter function

$Q(V)=K \ln \left(1+\mathrm{e}^{a\left(V-V_{0}\right)}\right)$

where $Q$ is the total charge and $V$ the operating voltage. The fit of the experimental data shown in Fig. 2 gives

$K=16.4 \mathrm{pC}$

$V_{0}=9.82 \mathrm{kV}$

$a=3.92 \times 10^{-3} \mathrm{~V}^{-1}$.

It should be stressed that the use of the Logistic function, which was introduced only on the ground of the functional behavior of $Q(V)$, turns out to be surprisingly accurate in accounting for a large number of experimental points with a 3 parameter fit. We notice also that the value of the parameter $a$ resulting from the fit, agrees within $20 \%$ with the one already found in Ref. [4] $(4.9 \pm 0.2 \%)$ based on the measurement of the average prompt charge of a similar but not identical gas. 


\section{Logistic avalanche model}

The Logistic equation is obtained by the lowest order non-linear correction to the usual exponential growth equation

$\frac{\mathrm{d} N}{\mathrm{~d} x}=\alpha N-\beta N^{2}$

where the constant parameters $\alpha$ and $\beta$ are the "generation" and the "mortality" rate factors, respectively. In the case of the avalanche growth $\alpha$ and $\beta$ mean the rate at which the free electrons are created and lost from the active avalanche front. The free running variable $x$ in Eq. (5) represents system evolution parameters such as the applied field or the avalanche path. We can rewrite the above equation in the form

$\frac{\mathrm{d} N}{\mathrm{~d} x}=\alpha N\left(1-\frac{N}{K}\right)$

$K=\frac{\alpha}{\beta}$

showing clearly that the growth rate tends to zero as $N$ reaches $K$. This is a stable stationary point whatever the initial conditions are. The case $\beta=0$ for a given $\alpha$ gives $K=\infty$ and corresponds to an exponential and unlimited growth. Integration of Eq. (6) gives the logistic function

$N(x)=\frac{K}{1+C K \mathrm{e}^{-\alpha x}}$

where $C$ depends on the initial population $N(0)$. If this last corresponds to a population above $K$, the solution decreases until $K$ is reached. In Fig. 3 are

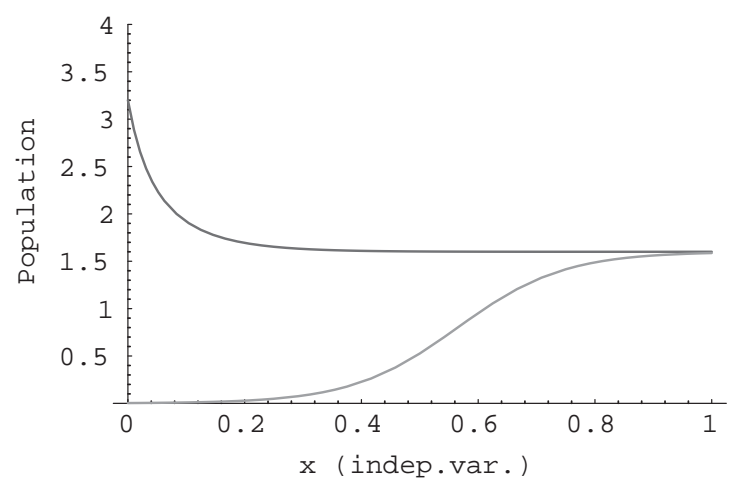

Fig. 3. Solutions of the logistic equation for $N(0)>K$ (upper curve), and $N(0)<K$ (lower curve). shown the two possible branches of the solution, for initial conditions corresponding to $N(0)>K$ (upper curve), and $N(0)<K$ (lower curve).

We note here an important feature of this kind of equation [9]: any power law on the right end of Eq. (5), growing more than linearly, brings the system to a stationary point. ${ }^{1}$ The quadratic nonlinear term means that the non-linear effect increases linearly with $N$ and was chosen as the simplest case giving the wanted behavior. It has also a simple physical meaning, in the avalanche case, being proportional to the probability that any of the electrons interacts with any of the others.

This behavior brings to the introduction of an useful concept for the avalanche description: the "active" avalanche, of charge $Q_{\text {act }}$, responsible for the creation of further ionization. $Q_{\text {act }}$ is a fraction of $Q_{\text {free, }}$ the total free electron charge including all the other electrons located in regions of lower field, due to the space charge effect. It is clear that in the exponential growth the total population is "active", while in the present case a saturation of the active population to the value $K$ is needed to reproduce the observed growth. This implies that the other electrons follow without multiplication, until they are collected at the anode or are attached by electronegative molecules. In this picture the saturation is reached when the number of electrons produced by each "generation" equals the number of electrons "left behind", so that the "active" population is left constant.

\section{Drift space vs. field saturation}

The growth of the avalanche is usually described with respect to the space variable representing the avalanche path. In the experimental case of the RPC, represented in Fig. 2, the drift space is fixed for each avalanche while the evolution of the total collected charge is observed for different values of the applied electric field, which is considered as the

\footnotetext{
${ }^{1}$ As the power of the non-linear term increases there are other stationary points contemporary possible (a so-called bifurcation), but now we look at the only real and positive one [9].
} 


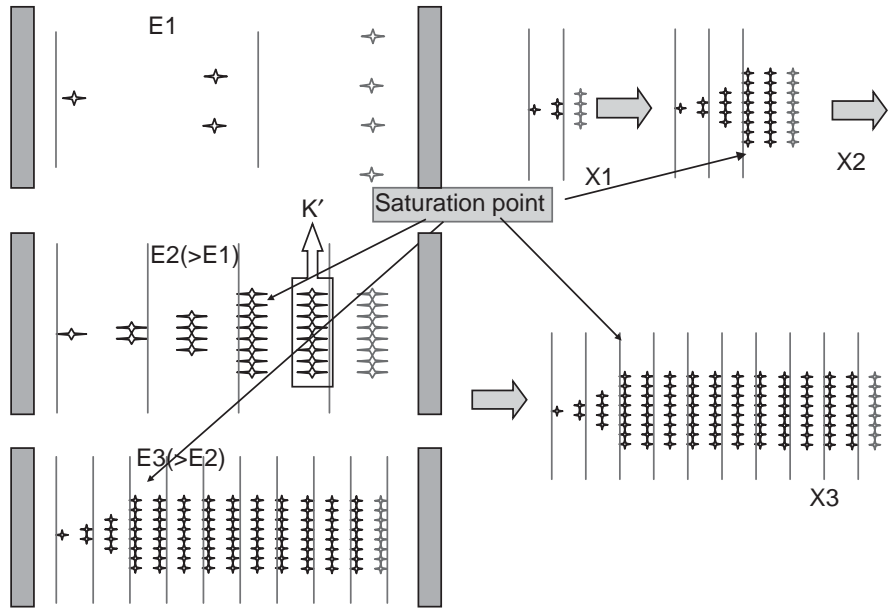

Fig. 4. Comparison of the avalanche evolution with respect to the electric field and the drift space. In a fixed gap (left) the larger is the field the earlier is the saturation.

independent variable. The avalanche evolution with respect to the electric field and the drift space are compared in Fig. 4.

The Eq. (6) explicitly written in terms of the applied voltage $V$ is

$$
\begin{aligned}
& \frac{\mathrm{d} Q_{\mathrm{act}}}{\mathrm{d} V}=a Q_{\mathrm{act}}\left(1-\frac{Q_{\mathrm{act}}}{K^{\prime}}\right) \\
& Q_{\mathrm{act}}(V)=\frac{K^{\prime}}{1+\mathrm{e}^{-a\left(V-V_{0}\right)}}
\end{aligned}
$$

where $Q_{\text {act }}$ is the charge carried out by the active electron population, $K^{\prime}$ is the charge of the limit population and depends on the gas gap width. $V_{0}$ is the field corresponding to $Q_{\text {act }}=K / 2$ and depends on the primary ionization $Q_{0}$ and on the gas properties; $a$ is the growth per unit field. The total collected charge at the end of the gas gap, as a function of the applied field, is the integral on the field variable of the active charge multiplied by the growth factor $a$

$$
\begin{aligned}
Q_{\mathrm{tot}}(V) & =Q_{0}+\int_{0}^{V} Q_{\mathrm{act}}\left(V^{\prime}\right) a \mathrm{~d} V^{\prime} \\
& =Q_{0}+K^{\prime} \ln \frac{1+\mathrm{e}^{a\left(V-V_{0}\right)}}{1+\mathrm{e}^{-a V_{0}}} .
\end{aligned}
$$

We can try to reformulate Eq. (5) in terms of drift space (or time), instead of voltage, as the evolution variable. If we suppose a linear relationship between the field and the drift space: $a \Delta V=$ $\alpha \Delta x$. This corresponds to the fact that the number of electron ionization lengths contained in a given drift space at a given electric field are the same in a bigger drift space with a proportionally lower field. Eqs. (9) and (11) can be easily rewritten in an identical form to describe the avalanche running along the lines of a fixed external electric field

$$
\begin{aligned}
& \frac{\mathrm{d} Q_{\mathrm{act}}}{\mathrm{d} x}=\alpha Q_{\mathrm{act}}\left(1-\frac{Q_{\mathrm{act}}}{K}\right), \quad Q_{\mathrm{act}}(x=0)=Q_{0} \\
& Q_{\mathrm{act}}(x)=\frac{K}{1+\mathrm{e}^{-\alpha\left(x-x_{0}\right)}}
\end{aligned}
$$

where $\alpha=\alpha(V)$ is the multiplication factor per unit length related to the externally fixed electric field value. We suppose here that the avalanche growth is dominated by a single primary cluster produced in proximity to the cathode surface. A more accurate calculation taking into account the primary ionization distribution in the gas gap, is given in Ref. [6]. The total produced charge after a path $x$ is the integral of the active charge multiplied by the growth factor $\alpha$, along the path

$$
\begin{aligned}
Q_{\mathrm{tot}}(x) & =Q_{0}+\int_{0}^{x} Q_{\mathrm{act}}\left(x^{\prime}\right) \alpha \mathrm{d} x^{\prime} \\
& =Q_{0}+K \ln \frac{1+\mathrm{e}^{\alpha\left(x-x_{0}\right)}}{1+\mathrm{e}^{-\alpha x_{0}}} .
\end{aligned}
$$

The linear approximation can be justified by the fact that the typical detector operative field range 
is small with respect to the applied field. $K$ and $K^{\prime}$ should coincide since they represent limit values of the same physical object, the "active" avalanche viewed into two different pictures. In this case $K$ does not depend on field and drift space but only on the gas properties. The mathematical details of this item will be developed in a forthcoming work. The above calculation refers to the case of negligible attachment. Otherwise all the above formulas are valid provided $\alpha$ is substituted with $\alpha^{*}=\alpha-\gamma$ where $\gamma$ is the attachment coefficient.

\section{The $Q_{\text {tot }}$ to $Q_{\text {prompt }}$ ratio}

We predict here the behavior of an observable, deeply dependent on the charge production dynamics, which is the ratio $Q_{\text {tot }} / Q_{\text {prompt }}$ of the total charge delivered in the gas to the prompt charge induced on the pickup electrode. In order to perform a test of the model this is compared to the results of the measurement described in Section 6 .

The $Q_{\text {tot }}$ to $Q_{\text {prompt }}$ ratio represents the efficiency of the fast signal production with respect to the total charge delivered in the process. In a pure exponential regime this ratio is $\alpha g$ for negligible electrode thickness, [10] $\alpha$ being the first Townsend coefficient and $g$ the gap width. Since $\alpha$ increases with the electric field the ratio also increases, indicating that the charge is mostly produced near to the end of the gas gap.

$Q_{\text {prompt }}$ is due to the motion of the free electrons $Q_{\text {free }}$ which include the active electrons $Q_{\text {act }}$ and the "inactive" ones until they are attached. According to the usual definition of the attachment coefficient $\gamma$ and from Eqs. (15) and (14)

$\frac{\mathrm{d} Q_{\text {free }}}{\mathrm{d} x}=\alpha Q_{\mathrm{act}}-\gamma Q_{\text {free }}$

$Q_{\text {prompt }}=\frac{1}{g} \int_{0}^{g} Q_{\text {free }}(x) \mathrm{d} x$

$R_{Q}=\frac{Q_{\text {tot }}}{Q_{\text {prompt }}}=g \frac{\int_{0}^{g} Q_{\text {act }}(x) \alpha \mathrm{d} x}{\int_{0}^{g} Q_{\text {free }}(x) \mathrm{d} x}$

where the last is the general expression for the ratio. For a completely saturated avalanche, i.e.
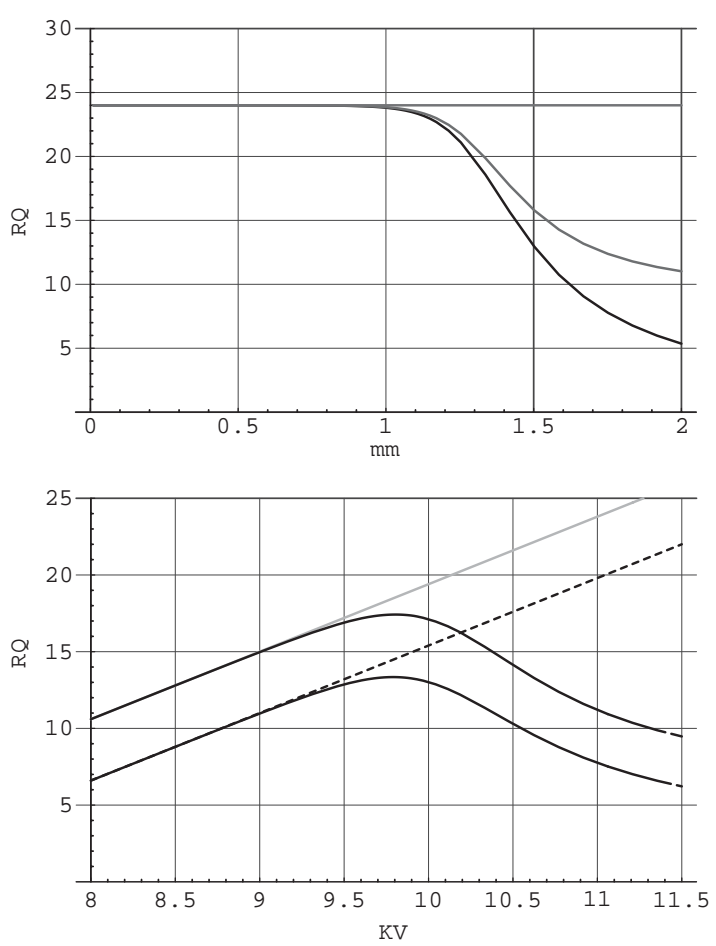

Fig. 5. Total to prompt charge ratio $R_{Q}$ vs. drift space (upper plot) and vs. applied voltage (lower plot), for the exponential model (straight lines) and the logistic model. In each set of curves the lower ones correspond to $\gamma=0$, and the upper one include the attachment with $\gamma=7 \mathrm{~mm}^{-1}$ (upper plot) and $\gamma=$ $2 \mathrm{~mm}^{-1}$ lower plot.

$x \gg x_{0}$, the Eq. (14) gives $Q_{\text {tot }}(x) \simeq K \alpha x$ and $R_{Q}$ is found to decrease down to the limit value 2 (for the case $\gamma=0 \Rightarrow Q_{\text {free }}=Q_{\text {tot }}$ ). In Fig. 5, upper plot, this ratio is calculated as a function of the drift space for $\gamma=0$ and $7 \mathrm{~mm}^{-1}$, and compared to the purely exponential case. In the lower plot it is represented as a function of the applied voltage on a fixed gap. The parameters of the calculation were fixed from the fit in Fig. 2. The various curves are referred to different determinations of $\alpha^{*}(V, \gamma)$.

\section{Measurement of $Q_{\text {tot }} / Q_{\text {prompt }}$}

This measurement is performed using a $30 \times$ $3 \mathrm{~cm}^{2}$ RPC with a single strip readout, one end connected to a $5 \mathrm{G}$ sample/s digital oscilloscope and the other terminated on its own impedance, 
Plot of Qtot and Qtot to Qprompt ratio vs. Qprmpt

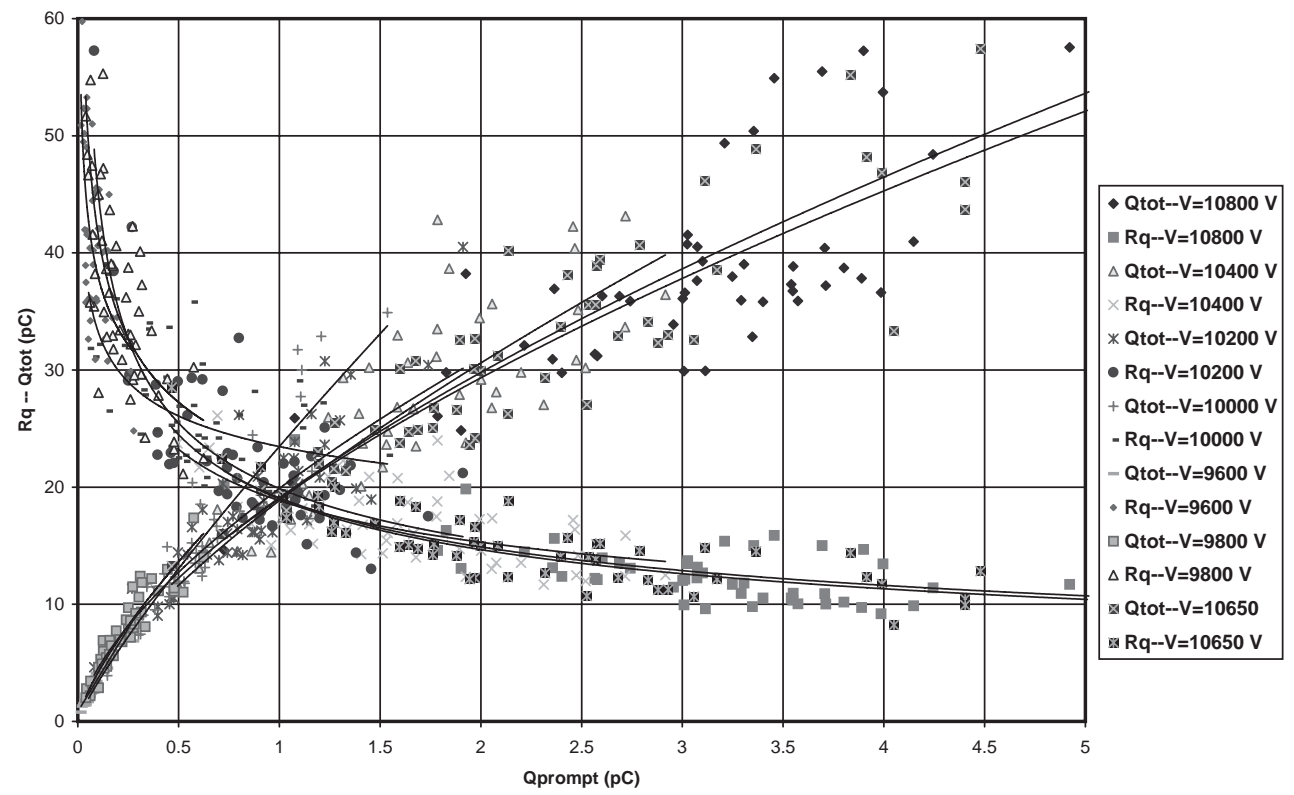

Qtot and Rq vs. Qprompt for different applied voltages

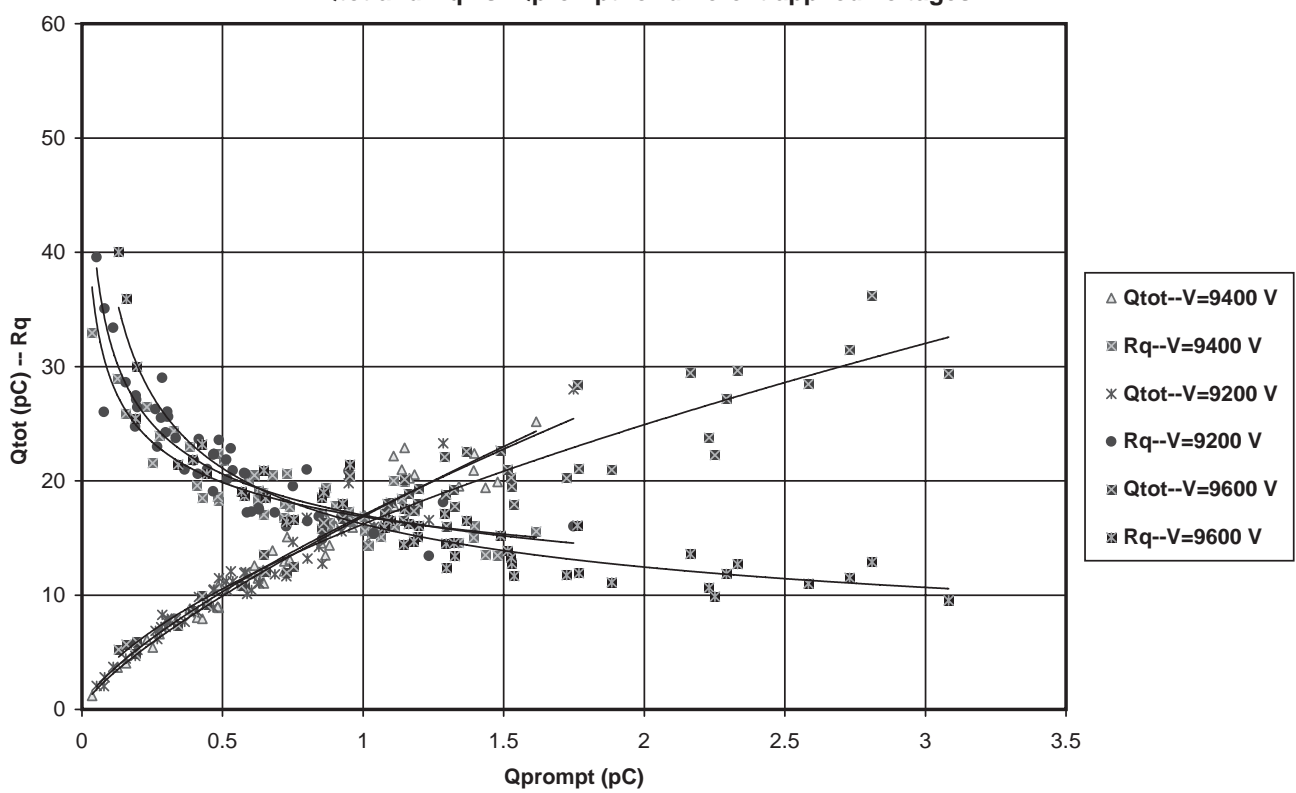

Fig. 6. $Q_{\text {tot }}$ and Ratio vs. $Q_{\text {prompt }}$ for the standard mixture (upper plot) and for the binary mixture without the $\mathrm{SF}_{6}$ lower plot. The different series refers to different applied voltages. Each black line is the generic power law trend line of each series.

which detects cosmic rays triggered by a three-fold scintillator coincidence. The RPC was operated in avalanche mode with a three component gas mixture $\mathrm{C}_{2} \mathrm{H}_{2} \mathrm{~F}_{4} / \mathrm{i}-\mathrm{C}_{4} \mathrm{H}_{10} / \mathrm{SF}_{6}=96.7 / 3 / .3$ and with the binary mixture without the $\mathrm{SF}_{6}$. The signal waveforms were used to measure the prompt charge whereas the ionic charge pulse [6] was readout connecting a second digital oscillo- 
scope across a shunt resistor placed in series to the ground graphite electrode. The total charge is the sum of the prompt electron charge and the ionic charge [10]. In order to avoid a non-uniform signal attenuation a conductive silver paint strip was placed on each graphite electrodes parallel to the long side of the plate (outside of the active area).

We recorded, for each voltage and gas condition, a few tens of avalanche waveforms which allowed to measure simultaneously the prompt and the total charge for each detected cosmic ray signal. The scatter plot of $Q_{\mathrm{tot}}$ and of $R_{Q}$ vs. $Q_{\text {prompt }}$ are reported in Fig. 6. The measured values of the prompt charge are corrected to take into account the attenuation due to the system geometry and electrode properties [10].

We notice that the points belonging to sets of different applied voltages dispose themselves along the same trend line. This indicates that the represented quantities do not explicitly depend on the applied voltage but on the avalanche saturation degree which is given by both the applied field and the primary ionization distribution inside the gas gap. The lower limit of $R_{Q}$, which is determined by the signal to noise ratio of the apparatus, is about 55 for the 3 component mixture and about 40 for the binary gas. The upper limit, determined by the avalanche to streamer transition, is barely above 10 in both cases, but corresponds to different prompt charge values. The comparison made for the same $Q_{\text {prompt }}$ shows that the value of $R_{Q}$ is about $20-30 \%$ larger for the 3 component gas, due to the $\mathrm{SF}_{6}$ attached electrons, which do not participate to the prompt signal production.

In order to compare these data to the theoretical predictions of the logistic model we report in Fig. 7 the average value of the $R_{Q}$ measurements vs. the applied voltage. The prediction of the present model is also reported for comparison. The exponential model is certainly excluded from this plot in the explored range of avalanche development, since it predicts an increasing value of $R_{Q}=$ $\alpha(V) g$. On the other side the $R_{Q}$ experimental curve shape is qualitatively compatible to the descending part of the logistic model prediction, represented in Fig. 5, in particular for the higher voltages. We do not observe any trace of the linear

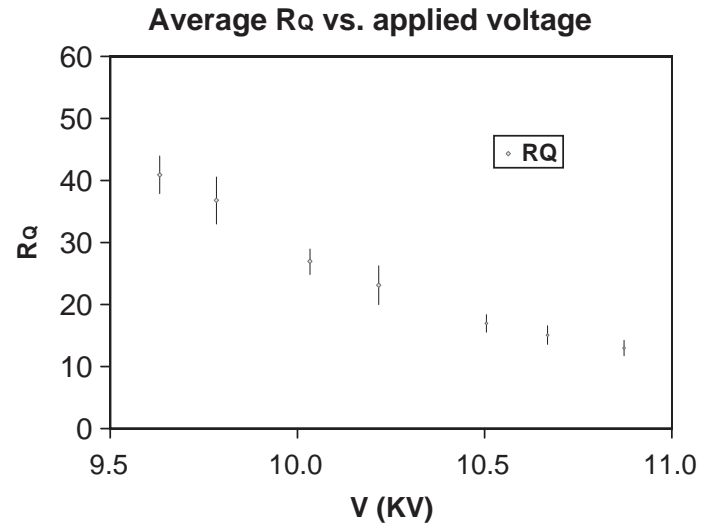

Fig. 7. Average value of $R_{Q}$ vs. the applied voltage for the 3 component gas. The behavior of experimental points exclude the purely exponential model.

increasing part of Fig. 5, that should be the signature of proportional regime, in the present voltage range.

\section{Conclusions}

In this paper, we proposed a natural structure in terms of which the description of a saturating avalanche is particularly meaningful and simple: the active avalanche being the electrons that can generate new free electrons. In the saturated regime the active avalanche moves along the electric field continuously creating new electrons which replace the ones that are left behind in lower field regions. We pointed out some important features:

- The introduced saturation mechanism does not depend directly on the attachment $\gamma$, that acts as a negative linear term in the rate differential equation 5 , but on the space charge screening effect.

- The attachment influences the prompt charge, determining how long a free electron can drift.

- The saturation occurs when the applied electric field is screened by the proper charge density produced by the same number of electrons $K$. This implies that for field saturated avalanches the electron density have to increase to balance the increasing field. 
- We notice that a saturated growth depends weakly on the initial conditions, in particular in Ref. [6] is shown that the total charge depends logarithmically on the primary cluster size.

In conclusion, we introduced for the first time the simplest non-linear growth differential equation, the Logistic Equation, to describe the saturation process in electron avalanches. The introduction of the $\mathrm{SF}_{6}$ in the gas mixture allowed to measure [5] the saturated avalanche total charge in a wide field region, retarding the streamer appearance, thus permitting an extensive test of the Logistic model with the presented $R_{Q}$ measurement method.

\section{References}

[1] H. Raether, Electron avalanches and breakdown in gases, Butterworth, London, 1964, pp. 78-88 (Section 3.7).

[2] P. Fonte, Nucl. Instr. and Meth. A 456 (2000) 6.

[3] G. Nicolis, I. Prigogine, Exploring Complexity. An Introduction, 1987.

[4] R. Cardarelli, et al., Nucl. Instr. and Meth. A 382 (1996) 470.

[5] P. Camarri, et al., Nucl. Instr. and Meth. A 414 (1998) 317.

[6] G. Aielli, Ph.D. Thesis, February 2001.

[7] G. Aielli, et al., Nucl. Instr. and Meth. A 456 (2000) 82.

[8] Funzione Logistica, Enciclopedia delle scienze Fisiche Treccani, Vol. VII.

[9] E. Ott, Chaos in Dynamical Systems, Cambridge University Press, Cambridge, MA, 1993.

[10] R. Santonico, Contributed paper to RPC 95 (Roma, October 11-12, 1995); Proceedings published on Scientifica Acta XI (1), pp. 1-10. 